\title{
The Effect of Added Weight on Foot Anthropometry in Pregnant Women and Controls \\ ${ }^{1}$ McCrory, JL, FASCM, ${ }^{1}$ Harrison, KD, ${ }^{2}$ Mancinelli, CA, ${ }^{3}$ Meszaros, $\mathrm{P}$ and ${ }^{2}$ Thomas \\ K. ${ }^{1}$ Division of Exercise Physiology, ${ }^{2}$ Division of Physical Therapy, ${ }^{3}$ Department of \\ Obstetrics and Gynecology, West Virginia University, Morgantown, WV
}

Foot anthropometry is altered by pregnancy, but it is not known if these changes are due to increased weight or hormonal effects of pregnancy on the body. The purpose of this study was to examine the effect of added weight on foot anthropometry in pregnant women and never-pregnant controls. Methods: Fifteen primigravid women and 13 nulliparous controls participated. Controls were matched to the pregnant women based on the pregnant women's self-reported pre-pregnancy weight. Informed consent was obtained. Data were collected on the pregnant subjects in each trimester and post-partum. Foot length, foot width, arch index, arch height index, arch rigidity index, and arch drop were assessed. To determine the effect of added weight on foot anthropometry, pregnant subjects in their first two trimesters donned a weighted pack on the anterior trunk such that total weight difference from pre-pregnancy weight was $124 \mathrm{~N}$, which was based on data from a previous study. Foot measurements were then repeated while the subjects wore this pack. Third trimester subjects did not wear a pack as they were at fullpregnancy weight. For post-partum subjects, their body weight plus the weight of the pack equaled their third trimester weight. For control subjects, their body weight plus the weight of the pack equaled the third trimester weight of the pregnant subject to whom they were matched. A MANOVA was performed with the independent variables of trimester (control, $1^{\text {st }}, 2^{\text {nd }}, 3^{\text {rd }}$, and post-partum) and weight condition (natural or weighted). Tukey post-hoc analyses were performed if appropriate $(\alpha=0.05)$. Results: Arch drop increased by $18 \%(\mathrm{p}=0.001)$ and arch rigidity index decreased by $1 \%$ $(\mathrm{p}=0.002)$ in the weighted condition compared to the natural condition. Increase in foot length and width and decrease in AHI with added weight was greater in pregnant subjects vs controls, with the change increasing over the course of the pregnancy. $(\mathrm{p}<0.05)$.

Conclusions: Adding weight produced significant changes in arch drop and arch rigidity index, although weight plus advancing pregnancy was related to further alterations in foot anthropometry, indicating that other factors, such as increased hormone concentrations, may play a role in foot anthropometry changes in pregnancy. 